Iranian Journal of Mathematical Sciences and Informatics

Vol. 15, No. 1 (2020), pp 111-124

DOI: 10.21859/IJMSI.15.1.111

\title{
Extensions of Some Fixed Point Theorems for Weak-Contraction Mappings in Partially Ordered Modular Metric Spaces
}

\author{
Karim Chaira $^{a}$, Abderrahim Eladraoui ${ }^{b *}$, Mustapha Kabil $^{c}$ \\ ${ }^{a}$ CRMEF, Rabat-Sale-Zemmour-Zaer, Street Allal El Fassi, Bab Madinat Al \\ Irfane, 6210,10000 Rabat, Morocco. \\ ${ }^{b}$ Laboratory of Algebra, Analysis and Applications, Faculty of Sciences Ben \\ M'Sick, Hassan II University of Casablanca, Morocco \\ ${ }^{c}$ Laboratory of Mathematics and Applications, Faculty of Sciences and \\ Technologies, Mohammedia, Hassan II University of Casablanca, Morocco.

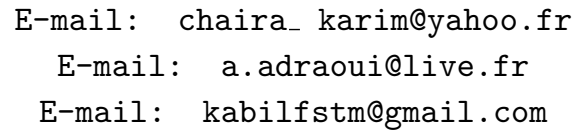

\begin{abstract}
The purpose of this paper is to establish fixed point results for a single mapping in a partially ordered modular metric space, and to prove a common fixed point theorem for two self-maps satisfying some
\end{abstract} \\ weak contractive inequalities.
}

Keywords: Fixed point, Weak contraction, Partially ordered space, Modular metric space.

2010 Mathematics Subject Classification: 46A80, 47H10.

\section{INTRODUCTION}

Many generalizations and extensions of Banach contraction principle have been studied in various settings (see [12, 21, 20, 22]). Most established results provide sufficient conditions for the existence and uniqueness of fixed points of

${ }^{*}$ Corresponding Author

Received 12 Jun 2017; Accepted 29 May 2019

(C)2020 Academic Center for Education, Culture and Research TMU

111 
certain classes of self-mappings (see [6, 7, 10, 24, 26, 27, 28]) and some of them provide iterative schemes and numerical algorithms to approximate those fixed points (see $[23,25,29])$.

In 2008, Dutta and Choudhury in [11] proved the following theorem, by using the weak contraction introduced by Alber and Guerre-Delabrere in [5].

Theorem 1.1. Let $(X, d)$ be a complete metric space, and let $T: X \rightarrow X$ be a self-mapping satisfying the following inequality:

$$
\psi(d(T x, T y)) \leq \psi(d(x, y))-\varphi(d(x, y)), \quad \text { for all }(x, y) \in X^{2}
$$

where $\psi, \varphi:[0,+\infty[\rightarrow[0,+\infty[$ are both continuous and nondecreasing functions with $\psi(t)=\varphi(t)=0$ if and only if $t=0$. Then $T$ has a unique fixed point.

In 2012, Abkar and Choudhury in [1] proved the following theorem which is a generalization of the above result in a partially ordered metric space having the following property $(\mathrm{P})$ :

for each non-decreasing sequence $\left(x_{n}\right)_{n \in \mathbb{N}} \subset X$ that converges to some $x \in X$, we have: $x_{n} \preceq x$ for all $n \in \mathbb{N}$.

Theorem 1.2. Let $(X, \preceq, d)$ be an ordered complete metric space with a partial order " $\preceq$ " and having the property $(P)$. Let $S, T: X \rightarrow X$ be two self mappings such that for all comparable $x, y \in X$,

$$
\psi_{1}(d(S x, T y)) \leq \psi_{2}(M(x, y))-\varphi(M(x, y))
$$

where

$$
M(x, y)=\max \left\{d(x, y), d(x, S x), d(y, T y), \frac{1}{2}(d(x, T y)+d(y, S x))\right\},
$$

$\psi_{1}, \psi_{2}:[0,+\infty[\rightarrow[0,+\infty[$ are both continuous and monotone non-decreasing functions and $\varphi:[0,+\infty[\rightarrow[0,+\infty[$ is lower semi-continuous function which satisfies $\psi_{1}(t)-\psi_{2}(t)+\varphi(t)>0$, for all $t>0$.

If there exists a point $x_{0} \in X$ satisfying

$$
x_{0} \preceq S x_{0} \preceq T S x_{0} \preceq S T S x_{0} \preceq(T S)^{2} x_{0} \preceq \cdots
$$

then there exists a point $u \in X$ such that $S u=T u=u$.

On the other hand, in 2010, Chistyakov in [8] and [9] has introduced the concept of modular metric space. This is a generalization of the classical modular spaces like Orlicz spaces (see [16]). Fixed point theorems in modular function spaces, generalizing the classical Banach fixed point theorem in metric spaces, have been studied extensively ( see $[4,6,17,18,24]$ ).

In recent years, there has been a great interest in the study of the fixed point property in modular metric spaces ( see $[2,3,19]$ ). For more details on modular metric fixed point theory, the reader may consult the books $[16,8]$.

In this paper we prove some fixed and common fixed point theorems for a weak contractive mapping in modular metric spaces. Our results generalize 
and extend the above theorems in partially ordered modular metric spaces for mappings satisfying weak contraction that involves three control functions.

\section{Preliminaries}

Let $X$ be a nonempty set. For a function $\omega:] 0,+\infty[\times X \times X \rightarrow[0,+\infty]$, we will use the notation

$$
\omega_{\lambda}(x, y)=\omega(\lambda, x, y), \quad \text { for all } \lambda>0 \text { and } x, y \in X .
$$

Definition 2.1. ([9]) A function $\omega:] 0,+\infty[\times X \times X \rightarrow[0,+\infty]$ is said to be modular metric on $X$ if it satisfies the following conditions:

(i) Given $x, y \in X, x=y$ if and only if $\omega_{\lambda}(x, y)=0$ for all $\lambda>0$;

(ii) For all $x, y \in X$, for all $\lambda>0, \omega_{\lambda}(x, y)=\omega_{\lambda}(y, x)$;

(iii) For all $x, y, z \in X$ and for all $\lambda, \mu>0, \omega_{\lambda+\mu}(x, y) \leq \omega_{\lambda}(x, z)+\omega_{\mu}(z, y)$.

In this case, $(X, \omega)$ is called modular metric space.

The modular $\omega$ is said to be regular if the condition (i) holds for some $\lambda>0$.

The modular $\omega$ is said to be convex if for all $\lambda, \mu>0$ and $x, y, z \in X$, we have:

$$
\omega_{\lambda+\mu}(x, y) \leq \frac{\lambda}{\lambda+\mu} \omega_{\lambda}(x, z)+\frac{\mu}{\lambda+\mu} \omega_{\mu}(z, y) .
$$

Note that for a modular metric $\omega$ on a set $X$, and any $x, y \in X$, the function $\lambda \rightarrow \omega_{\lambda}(x, y)$ is non-increasing on $] 0,+\infty[$. Indeed, if $0<\mu<\lambda$, then

$$
\omega_{\lambda}(x, y) \leq \omega_{\lambda-\mu}(x, x)+\omega_{\mu}(x, y)=\omega_{\mu}(x, y) .
$$

Definition 2.2. ([8]) Let $(X, \omega)$ be a modular metric space. Fix $x_{0} \in X$. Set

$$
X_{\omega}=X_{\omega}\left(x_{0}\right)=\left\{x \in X: \omega_{\lambda}\left(x, x_{0}\right) \longrightarrow 0 \text { as } \lambda \longrightarrow \infty\right\},
$$

and

$$
X_{\omega}^{*}=X_{\omega}^{*}\left(x_{0}\right)=\left\{x \in X: \exists \lambda>0, \omega_{\lambda}\left(x, x_{0}\right)<\infty\right\} .
$$

The two linear spaces $X_{\omega}$ and $X_{\omega}^{*}$ are said to be modular spaces (around $x_{0}$ ).

Note that $X_{\omega}$ is metrizable by the metric

$$
d_{\omega}(x, y)=\inf \left\{t>0: \omega_{t}(x, y) \leq t\right\} .
$$

If $\omega$ is convex, then $X_{\omega}^{*}=X_{\omega}$ and we can endowed these sets with the metric $d_{\omega}^{*}$ defined by

$$
d_{\omega}^{*}(x, y)=\inf \left\{t>0: \omega_{t}(x, y) \leq 1\right\} .
$$

Definition 2.3. ([2]) Let $\omega$ be a modular metric on $X$.

(1) We say that a sequence $\left\{x_{n}\right\} \subset X_{\omega}$ is $\omega$-convergent to some $x \in X_{\omega}$ if and only if $\lim _{n \rightarrow+\infty} \omega_{1}\left(x_{n}, x\right)=0$. We will call $x$ the $\omega$-limit of $\left\{x_{n}\right\}$.

(2) We say that a sequence $\left\{x_{n}\right\} \subset X_{\omega}$ is $\omega$-Cauchy if

$$
\lim _{n, m \rightarrow+\infty} \omega_{1}\left(x_{n}, x_{m}\right)=0 .
$$


(3) We say that $M \subset X_{\omega}$ is $\omega$-closed if the $\omega$-limit of an $\omega$-convergent sequence of $M$ is in $M$.

(4) We say that $M \subset X_{\omega}$ is $\omega$-complete if any $\omega$-Cauchy sequence in $M$ is $\omega$-convergent and its $\omega$-limit belongs to $M$.

(5) We say that $\omega$ satisfies Fatou property if we have

$$
\omega_{1}(x, y) \leq \liminf _{n \rightarrow+\infty} \omega_{1}\left(x_{n}, y\right)
$$

for any sequence $\left\{x_{n}\right\} \subset X_{\omega}$ which $\omega$-converges to $x$ and for any $y \in X_{\omega}$.

Definition 2.4. ([8]) Let $\omega$ be a modular metric on $X$. We say that $\omega$ satisfies the $\Delta_{2}$-condition, or simply $\omega$ is $\Delta_{2}$, if, given a sequence $\left\{x_{n}\right\} \subset X_{\omega}, x \in X_{\omega}$ and $\lambda>0$ such that $\lim _{n \rightarrow+\infty} \omega_{\lambda}\left(x_{n}, x\right)=0$, we have $\lim _{n \rightarrow+\infty} \omega_{\frac{\lambda}{2}}\left(x_{n}, x\right)=0$.

Definition 2.5. Let $\omega$ be a modular metric on $X$. We say that $\omega$ satisfies the $\Delta_{2}$-type condition if there exists a constant $K>0$ such that

$$
\omega_{\frac{\lambda}{2}}(x, y) \leq K \omega_{\lambda}(x, y),
$$

for all $x, y \in X_{\omega}$ and any $\lambda>0$.

The following results are immediate:

Lemma 2.6. If $\omega$ satisfies the $\Delta_{2}-$ type condition, then $\omega$ satisfies the $\Delta_{2}-$ condition.

Lemma 2.7. Let $\left\{x_{n}\right\}$ be a sequence in $X_{\omega}$. Let $\lambda>0$. If $\omega$ satisfies the $\Delta_{2}-$ type condition, then $\left\{x_{n}\right\}$ is $\omega$-Cauchy if and only if $\lim _{n, m \rightarrow+\infty} \omega_{\lambda}\left(x_{n}, x_{m}\right)=0$.

Lemma 2.8. If $\omega$ satisfies the $\Delta_{2}-$ type condition, then $\omega$ is regular.

Lemma 2.9. If $\omega$ satisfies the $\Delta_{2}-$ type condition, then $\omega_{\lambda}(x, y)<\infty$, for all $\lambda>0$ and for all $(x, y) \in X_{\omega}^{2}$.

Proof. Suppose that there exists $\lambda>0$ and $x, y \in X_{\omega}=X_{\omega}\left(x_{0}\right)$ such that $\omega_{\lambda}(x, y)=\infty$. Since $\omega$ satisfies the $\Delta_{2}-$ type condition, then, for all $n \in$ $\mathbb{N}^{*}, \omega_{2^{n} \lambda}(x, y)=\infty$. Since $\omega_{2^{n} \lambda}(x, y) \leq \omega_{2^{n-1} \lambda}\left(x, x_{0}\right)+\omega_{2^{n-1} \lambda}\left(y, x_{0}\right)$ and $x, y \in X_{\omega}\left(x_{0}\right)$, we have: $\lim _{n \rightarrow+\infty} \omega_{2^{n-1} \lambda}\left(x, x_{0}\right)=0$ and $\lim _{n \rightarrow+\infty} \omega_{2^{n-1} \lambda}\left(y, x_{0}\right)=0$. Then, $\lim _{n \rightarrow+\infty} \omega_{2^{n} \lambda}(x, y)=0$. Which is a contradiction.

Lemma 2.10. Let $(X, \omega)$ be a modular space. Let $\left\{x_{n}\right\}$ be a sequence in $X_{\omega}$. If $\left\{x_{n}\right\}$ is not $\omega$-Cauchy, then there exists $\varepsilon>0$ and two subsequences of integers $\left\{n_{k}\right\}$ and $\left\{m_{k}\right\}$ such that $n_{k}>m_{k} \geq k, \omega_{1}\left(x_{n_{k}}, x_{m_{k}}\right) \geq \varepsilon$ and $\omega_{\frac{1}{2}}\left(x_{n_{k}-1}, x_{m_{k}}\right)<\varepsilon$.

Proof. If we suppose that $\left(x_{n}\right)_{n \in \mathbb{N}}$ is not a $\omega$-Cauchy, then there exists $\varepsilon>0$ and for all $k \in \mathbb{N}$ there exists $n_{k}, m_{k} \in \mathbb{N}$ such that $n_{k}>m_{k} \geq k$ and $\omega_{1}\left(x_{n_{k}}, x_{m_{k}}\right) \geq \varepsilon$. Let us fix $k \in \mathbb{N}$, and consider the set

$$
\mathcal{A}_{k}=\left\{h \in \mathbb{N}^{*}: h>m_{k} \geq k \text { and } \omega_{1}\left(x_{h}, x_{m_{k}}\right) \geq \varepsilon\right\} .
$$


Since $n_{k} \in \mathcal{A}_{k}$, then $\mathcal{A}_{k} \neq \emptyset$. Let us consider the set:

$$
\mathcal{B}_{k}=\left\{h \in \mathcal{A}_{k}: \omega_{\frac{1}{2}}\left(x_{h}, x_{m_{k}}\right) \geq \varepsilon\right\} .
$$

One can see that $\mathcal{B}_{k} \subseteq \mathbb{N}^{*}$ and $\mathcal{B}_{k} \neq \emptyset$. Since

$$
\omega_{\frac{1}{2}}\left(x_{n_{k}}, x_{m_{k}}\right) \geq \omega_{1}\left(x_{n_{k}}, x_{m_{k}}\right) \geq \varepsilon,
$$

then $\mathcal{B}_{k}$ admits the least element $n_{k}^{\prime}$ that belongs to $\mathcal{A}_{k}$, and so $n_{k}^{\prime}>m_{k} \geq k, \omega_{1}\left(x_{n_{k}^{\prime}}, x_{m_{k}}\right) \geq \varepsilon$ and $\omega_{\frac{1}{2}}\left(x_{n_{k}^{\prime}-1}, x_{m_{k}}\right)<\varepsilon$.

Using the same argument as in the proof of Lemma 2.10 and applying Lemma 2.7, we have the following:

Lemma 2.11. Let $s, t \in \mathbb{N}^{*}$. If $\omega$ satisfies the $\Delta_{2}-$ type condition and $\left\{x_{n}\right\}$ is not a $\omega$-Cauchy sequence, then there exists $\varepsilon>0$ and two subsequences of integers $\left\{n_{k}\right\}$ and $\left\{m_{k}\right\}$ such that $n_{k}>m_{k} \geq k, \omega_{2^{s}}\left(x_{n_{k}}, x_{m_{k}}\right) \geq \varepsilon$ and $\omega_{\frac{1}{2^{t}}}\left(x_{n_{k}-1}, x_{m_{k}}\right)<\varepsilon$.

Lemma 2.12. Let $(X, \omega)$ be a modular space such that $\omega$ is convex and satisfies the $\Delta_{2}$-condition. If $\left\{x_{n}\right\}$ is a sequence in $X_{\omega}$ such that $\lim _{n \rightarrow+\infty} \omega_{1}\left(x_{n}, x_{n+1}\right)=$ 0 , then $\left\{x_{n}\right\}$ is $\omega$-Cauchy.

Proof. Suppose that $\left\{x_{n}\right\}$ is not $\omega$-Cauchy, then according to Lemma 2.10, there exists $\varepsilon>0$ and two subsequences of integers $\left\{n_{k}\right\}$ and $\left\{m_{k}\right\}$ such that $n_{k}>m_{k} \geq k$ and $\omega_{1}\left(x_{n_{k}}, x_{m_{k}}\right) \geq \varepsilon$ and $\omega_{\frac{1}{2}}\left(x_{n_{k}-1}, x_{m_{k}}\right)<\varepsilon$. Since $\omega$ is convex, we have:

$$
\omega_{1}\left(x_{n_{k}}, x_{m_{k}}\right) \leq \frac{1}{2} \omega_{\frac{1}{2}}\left(x_{n_{k}-1}, x_{m_{k}}\right)+\frac{1}{2} \omega_{\frac{1}{2}}\left(x_{n_{k}-1}, x_{n_{k}}\right) .
$$

Then, for all $k \in \mathbb{N}$

$$
\varepsilon \leq \frac{\varepsilon}{2}+\frac{1}{2} \omega_{\frac{1}{2}}\left(x_{n_{k}-1}, x_{n_{k}}\right)
$$

Since $\omega$ satisfies the $\Delta_{2}$-type condition, then $\lim _{n \rightarrow+\infty} \omega_{\frac{1}{2}}\left(x_{n_{k}-1}, x_{n_{k}}\right)=0$. So, $\varepsilon \leq \frac{\varepsilon}{2}$. Which is a contradiction.

Definition 2.13. Let $X$ be a nonempty set. Then $(X, \preceq, \omega)$ is called a partially ordered modular metric space if and only if (i) $(X, \omega)$ is a modular metric space and (ii) $(X, \preceq)$ is a partially ordered set.

Definition 2.14. Let $(X, \preceq, \omega)$ be a partially ordered modular metric space. We say that $\omega$ satisfies the property $(\mathrm{P})$, if a non-decreasing sequence $\left\{x_{n}\right\}$ $\omega$-converges to some $x \in X_{\omega}$, then $x_{n} \preceq x$ for all $n \in \mathbb{N}$.

Definition 2.15. Let $C$ be a nonempty subset of $X_{\omega}$. A self-mapping $T: C \rightarrow$ $C$ is said to be $\omega$-continuous, if a sequence $\left\{x_{n}\right\} \omega$-converges to some $x \in C$, then $\left\{T x_{n}\right\} \omega$-converges to $T x$.

Definition 2.16. We say that a partially ordered set $(X, \preceq)$ is up-directed, if for all $(x, y) \in X^{2}$ there exists an element $z \in X$ such that $x \preceq z$ and $y \preceq z$. 
We will use the following notations:

Let $X$ be a nonempty set and $\mathrm{S}$ and $\mathrm{T}$ be a two self-mappings on $X$. We denote by $\mathfrak{F}(S)$ the fixed point set of S, i.e., $\mathfrak{F}(S):=\{x \in X: S x=x\}$. Also, we denote by $\mathfrak{F}(S, T)$ the common fixed point set of $\mathrm{S}$ and T, i.e., $\mathfrak{F}(S, T)=\mathfrak{F}(S) \cap \mathfrak{F}(T)$

\section{Main Results}

Let us consider three functions $\psi_{1}, \psi_{2}, \varphi: \mathbb{R}^{+} \rightarrow \mathbb{R}^{+}$such that:

(a) $\psi_{1}, \psi_{2}$ are continuous and $\varphi$ is lower semi-continuous.

(b) $\psi_{1}$ is strictly increasing.

(c) For all $i \in\{1,2\}, \psi_{i}(0)=\varphi(0)=0$.

(d) For all $t>0, \psi_{1}(t)-\psi_{2}(t)+\varphi(t)>0$.

3.1. Fixed point of $\left(\psi_{1}, \psi_{2}, \varphi\right)$-contraction. In this section, we obtain fixed point results for a single mapping satisfying a $\left(\psi_{1}, \psi_{2}, \varphi\right)$-contractive condition in the framework of a partially ordered modular metric space.

Theorem 3.1. Let $(X, \omega, \preceq)$ be a partially modular metric space. Assume that $\omega$ satisfies the $\Delta_{2}$-type condition. Let $C$ be an $\omega$-complete nonempty subset of $X_{\omega}$. Let $T: C \rightarrow C$ be a non-decreasing self-mapping. If the following conditions are verified

(i) for all comparable elements $x, y \in C$,

$$
\psi_{1}\left(\omega_{1}(T x, T y)\right) \leq \psi_{2}\left(\omega_{1}(x, y)\right)-\varphi\left(\omega_{1}(x, y)\right)
$$

(ii) there exists an element $x_{0} \in C$ such that $x_{0} \preceq T x_{0}$;

(iii) $\omega$ satisfies the property $(P)$,

then $T$ has a fixed point in $C$. Moreover if $(C, \preceq)$ is up-directed, then the fixed point is unique.

Proof. We divide this proof into four steps.

Step.1. Consider the sequence $\left\{x_{n}\right\}$ defined by $x_{n}=T^{n} x_{0}$. One can see that

$$
x_{n} \preceq x_{n+1}, \quad \text { for all } n \in \mathbb{N} \text {. }
$$

If we suppose that there exists an integer $n$ such that $x_{n}=x_{n+1}$, then $T$ admits at least a fixed point in $C$. So, let us assume that $x_{n} \neq x_{n+1}$ for all $n \in \mathbb{N}$.

In (i), if we take $x=x_{n}$ and $y=x_{n+1}$, we obtain:

$\psi_{1}\left(\omega_{1}\left(x_{n+1}, x_{n+2}\right)\right) \leq \psi_{2}\left(\omega_{1}\left(x_{n}, x_{n+1}\right)\right)-\varphi\left(\omega_{1}\left(x_{n}, x_{n+1}\right)\right) \leq \psi_{1}\left(\omega_{1}\left(x_{n}, x_{n+1}\right)\right)$

Then the sequence $\left\{\omega_{1}\left(x_{n}, x_{n+1}\right)\right\}$ is decreasing and bounded below. So, it converges to some nonnegative real $r$. If we suppose that $r>0$, then taking 
the limit superior as $n \rightarrow+\infty$ in the first part of the above inequality, we obtain

$\limsup _{n \rightarrow+\infty} \psi_{1}\left(\omega_{1}\left(x_{n+1}, x_{n+2}\right)\right) \leq \limsup _{n \rightarrow+\infty} \psi_{2}\left(\omega_{1}\left(x_{n}, x_{n+1}\right)\right)-\liminf _{n \rightarrow+\infty} \varphi\left(\omega_{1}\left(x_{n}, x_{n+1}\right)\right)$.

Using the continuity of $\psi_{1}$ and $\psi_{2}$ and the lower semi-continuity of $\varphi$ and applying the condition (d), we obtain

$$
\psi_{1}(r) \leq \psi_{2}(r)-\varphi(r)<\psi_{1}(r),
$$

a contradiction. Therefore, $r=0$.

Step.2. Let us prove that the sequence $\left\{x_{n}\right\}$ is $\omega$-Cauchy. Suppose that $\left\{x_{n}\right\}_{n \in \mathbb{N}}$ is not $\omega$-Cauchy. Then, according to Lemma 2.11 for $\omega_{4}$ and $\omega_{\frac{1}{2}}$, there exists $\varepsilon>0$ and subsequences of integers $\left\{m_{k}\right\}$ and $\left\{n_{k}\right\}$ such that

$$
m_{k}>n_{k} \geq k, \omega_{4}\left(x_{m_{k}}, x_{n_{k}}\right) \geq \varepsilon \text { and } \omega_{\frac{1}{2}}\left(x_{m_{k}-1}, x_{n_{k}}\right)<\varepsilon \text {. }
$$

In (i), if we take $x=x_{m_{k}-1}$ and $y=x_{n_{k}-1}$, and since $x_{n_{k}-1} \preceq x_{m_{k}-1}$, we obtain:

$$
\begin{aligned}
\psi_{1}(\varepsilon) \leq \psi_{1}\left(\omega_{4}\left(x_{m_{k}}, x_{n_{k}}\right)\right) & \leq \psi_{1}\left(\omega_{1}\left(x_{m_{k}}, x_{n_{k}}\right)\right) \\
& \leq \psi_{2}\left(\omega_{1}\left(x_{m_{k}-1}, x_{n_{k}-1}\right)\right)-\varphi\left(\omega_{1}\left(x_{m_{k}-1}, x_{n_{k}-1}\right)\right)
\end{aligned}
$$

And we have

$$
\varepsilon \leq \omega_{4}\left(x_{m_{k}}, x_{n_{k}}\right) \leq \omega_{1}\left(x_{m_{k}-1}, x_{n_{k}-1}\right)+\omega_{1}\left(x_{m_{k}-1}, x_{m_{k}}\right)+\omega_{2}\left(x_{n_{k}-1}, x_{n_{k}}\right),
$$

and

$$
\omega_{1}\left(x_{m_{k}-1}, x_{n_{k}-1}\right) \leq \omega_{\frac{1}{2}}\left(x_{m_{k}-1}, x_{n_{k}}\right)+\omega_{\frac{1}{2}}\left(x_{n_{k}-1}, x_{n_{k}}\right) .
$$

Thus

$\varepsilon-\omega_{1}\left(x_{m_{k}-1}, x_{m_{k}}\right)-\omega_{2}\left(x_{n_{k}-1}, x_{n_{k}}\right) \leq \omega_{1}\left(x_{m_{k}-1}, x_{n_{k}-1}\right) \leq \varepsilon+\omega_{\frac{1}{2}}\left(x_{n_{k}-1}, x_{n_{k}}\right)$.

Since $\omega$ satisfies the $\Delta_{2}$-type condition, then

$$
\lim _{k \rightarrow+\infty} \omega_{\frac{1}{2}}\left(x_{n_{k}-1}, x_{n_{k}}\right)=\lim _{k \rightarrow+\infty} \omega_{2}\left(x_{n_{k}-1}, x_{n_{k}}\right)=0 .
$$

So $\lim _{k \rightarrow+\infty} \omega_{1}\left(x_{m_{k}-1}, x_{n_{k}-1}\right)=\varepsilon$. And by letting $k \rightarrow+\infty$, in the inequality (3.3), we obtain

$$
\psi_{1}(\varepsilon) \leq \psi_{2}(\varepsilon)-\varphi(\varepsilon)<\psi_{1}(\varepsilon)
$$

which is a contradiction, then $\left\{x_{n}\right\}$ is $\omega$-Cauchy in $C$. Since $\mathrm{C}$ is $\omega$-complete, there exists $x \in C$ such that $\lim _{n \rightarrow+\infty} \omega_{1}\left(x_{n}, x\right)=0$.

Step.3. Let us prove that $x$ is a fixed point of $T$. Since $x_{n} \preceq x$, for all $n \in \mathbb{N}^{*}$, we have:

$$
\psi_{1}\left(\omega_{1}\left(x_{n}, T x\right)\right) \leq \psi_{2}\left(\omega_{1}\left(x_{n-1}, x\right)\right)-\varphi\left(\omega_{1}\left(x_{n-1}, x\right)\right) \leq \psi_{1}\left(\omega_{1}\left(x_{n-1}, x\right)\right) .
$$

Then $0 \leq \omega_{1}\left(x_{n}, T x\right) \leq \omega_{1}\left(x_{n-1}, x\right)$ and by passing to limit, we obtain

$$
\lim _{n \rightarrow+\infty} \omega_{1}\left(x_{n}, T x\right)=0 .
$$


And as

$$
\omega_{2}(x, T x) \leq \omega_{1}\left(x_{n}, x\right)+\omega_{1}\left(x_{n}, T x\right),
$$

then $\omega_{2}(x, T x)=0$ and since $\omega$ is regular, we obtain $T x=x$.

Step.4. Assume that $(C, \preceq)$ is up-directed. Let $y \in C$ be another fixed point of $T$. There exists $z \in C$ such that $x \preceq z$ and $y \preceq z$. As $T$ is non-decreasing, we have for all $n \in \mathbb{N}, x \preceq T^{n} z=z_{n}$ and $y \preceq T^{n} z=z_{n}$. Then

$$
\psi_{1}\left(\omega_{1}\left(x, z_{n+1}\right)\right) \leq \psi_{2}\left(\omega_{1}\left(x, z_{n}\right)\right)-\varphi\left(\omega_{1}\left(x, z_{n}\right)\right) \leq \psi_{1}\left(\omega_{1}\left(x, z_{n}\right)\right) .
$$

Thus the sequence $\left\{\omega_{1}\left(x, z_{n}\right)\right\}$ is decreasing and bounded below. So, it converges to some $l \geq 0$ and by tending $n$ to $+\infty$ in the above inequality, we obtain $l=0$. By the same argument we prove that $\lim _{n \rightarrow+\infty} \omega_{1}\left(y, z_{n}\right)=0$. By passing to limit in the following inequality

$$
\omega_{2}(x, y) \leq \omega_{1}\left(x, z_{n}\right)+\omega_{1}\left(y, z_{n}\right)
$$

we obtain $\omega_{2}(x, y)=0$. Since $\omega$ is regular, we have $x=y$. Then, $T$ admits a unique fixed point in $C$.

In what follows, we prove that Theorem 3.1 is still valid if we neglect the property $(\mathrm{P})$ and assume that $T$ is $\omega$-continuous.

Theorem 3.2. Let $(X, \preceq, \omega)$ be a partially modular metric space. Assume that $\omega$ satisfies the $\Delta_{2}$-type condition. Let $C$ be an $\omega$-complete nonempty subset of $X_{\omega}$. Let $T: C \rightarrow C$ be an $\omega$-continuous non-decreasing self-mapping. If the following conditions are verified

(i) for all comparable elements $x, y \in C$,

$$
\psi_{1}\left(\omega_{1}(T x, T y)\right) \leq \psi_{2}\left(\omega_{1}(x, y)\right)-\varphi\left(\omega_{1}(x, y)\right)
$$

(ii) there exists an element $x_{0} \in C$ such that $x_{0} \preceq T x_{0}$,

then $T$ has a fixed point in $C$. Moreover if $(C, \preceq)$ is up-directed, then the fixed point is unique.

Proof. Following the proof of Theorem 3.1 we only have to check that $T x=$ $x$. As $\left\{x_{n}\right\} \omega$-converges to $x$ and $T$ is $\omega$-continuous, then the sequence $\left\{x_{n+1}\right\}=\left\{T x_{n}\right\}$ is $\omega$-convergent to $T x$. And since the regularity of $\omega$ implies the uniqueness of limit, we obtain $T x=x$.

If we take $\psi_{1}=\psi_{2}=\psi$, and if we define $\omega$ by:

$$
\omega_{\lambda}(x, y)=\frac{d(x, y)}{\lambda} \text {, for all }(x, y) \in X^{2}
$$

we obtain the following result proved, in 2010, by Harjani and Sadarangani in the setting of metric spaces [13, Theorem 2.1 and Theorem 2.2]).

Corollary 3.3. Let $(X, \preceq)$ be a partially ordered set and suppose that there exists a metric $d$ on $X$ such that $(X, d)$ is a complete metric space. Let $T: X \rightarrow$ $X$ be a non-decreasing self-mapping. If the following conditions are verified 
(i) for all comparable elements $x, y \in C$,

$$
\psi(d(T x, T y)) \leq \psi(d(x, y))-\varphi(d(x, y))
$$

(ii) there exists an element $x_{0} \in C$ such that $x_{0} \preceq T x_{0}$;

(iii) $X$ satisfies the property $(P)$ or $T$ is $\omega$-continuous,

then $T$ has a unique fixed point in $C$ provided that $(C, \preceq)$ is up-directed.

3.2. Common fixed point of generalized $\left(\psi_{1}, \psi_{2}, \varphi\right)$-contraction. In this section, we obtain a common fixed point for a pair of mappings satisfying a generalized $\left(\psi_{1}, \psi_{2}, \varphi\right)$-contractive condition in the framework of a partially ordered convex modular metric space. We set

$$
M(x, y)=\max \left\{\omega_{1}(x, y), \omega_{1}(x, S x), \omega_{1}(y, T y), \omega_{2}(x, T y)+\omega_{2}(y, S x)\right\} .
$$

Theorem 3.4. Let $(X, \preceq, \omega)$ be a partially ordered modular metric space where $\omega$ is convex and satisfies the $\Delta_{2}$-type condition. Let $C$ be an $\omega$-complete nonempty subset of $X_{\omega}$ and $T, S: C \rightarrow C$ be two self-mappings. If the following conditions are verified:

(i) for all comparable elements $x, y \in C$,

$$
\psi_{1}\left(\omega_{1}(S x, T y)\right) \leq \psi_{2}(M(x, y))-\varphi(M(x, y)) ;
$$

(ii) $\omega$ satisfies Fatou property or $T$ is $\omega$-continuous;

(iii) there exists an element $x_{0} \in C$ such that

$$
x_{0} \preceq S x_{0} \preceq T S x_{0} \preceq S T S x_{0} \preceq(T S)^{2} x_{0} \preceq S(T S)^{2} x_{0} \preceq \ldots
$$

(iv) $\omega$ satisfies the property $(P)$,

then $S$ and $T$ have a common fixed point in $C$ and $\mathfrak{F}(S, T)=\mathfrak{F}(S)=\mathfrak{F}(T)$. Particulary, if $\mathfrak{F}(S, T)$ is totally ordered, then $T$ and $S$ have a unique fixed point.

Proof. Consider the sequence $\left\{x_{n}\right\}$ defined by

$$
x_{2 n+1}=S x_{2 n} \text { and } x_{2 n+2}=T x_{2 n+1}, \text { for all } n \in \mathbb{N} .
$$

The condition (ii) insures that $\left\{x_{n}\right\}$ is non-decreasing. If there exists an integer $n$ such that

$$
x_{2 n}=x_{2 n+1}=x_{2 n+2},
$$

then $x_{2 n}$ is a common fixed point of $S$ and $T$. Otherwise, suppose that

$$
x_{2 n} \neq x_{2 n+1} \text { or } x_{2 n} \neq x_{2 n+2} \text {, for all } n \in \mathbb{N} \text {. }
$$

Let $n \in \mathbb{N}$. From $x_{2 n} \preceq x_{2 n+1}$ and applying the inequality (3.6) for $x=x_{2 n}$ and $y=x_{2 n+1}$, we obtain

$$
\psi_{1}\left(\omega_{1}\left(x_{2 n+1}, x_{2 n+2}\right)\right) \leq \psi_{2}\left(M\left(x_{2 n}, x_{2 n+1}\right)\right)-\varphi\left(M\left(x_{2 n}, x_{2 n+1}\right)\right)
$$


where

$$
M\left(x_{2 n}, x_{2 n+1}\right)=\max \left\{\omega_{1}\left(x_{2 n}, x_{2 n+1}\right), \omega_{1}\left(x_{2 n+1}, x_{2 n+2}\right), \omega_{2}\left(x_{2 n}, x_{2 n+2}\right)\right\} .
$$

Since $\omega$ is convex, we have

$$
\omega_{2}\left(x_{2 n}, x_{2 n+2}\right) \leq \frac{1}{2}\left(\omega_{1}\left(x_{2 n}, x_{2 n+1}\right)+\omega_{1}\left(x_{2 n+1}, x_{2 n+2}\right)\right) .
$$

Then

$$
M\left(x_{2 n}, x_{2 n+1}\right)=\max \left\{\omega_{1}\left(x_{2 n}, x_{2 n+1}\right), \omega_{1}\left(x_{2 n+1}, x_{2 n+2}\right)\right\} .
$$

If we suppose that there exists an integer $n$ such that:

$$
\omega_{1}\left(x_{2 n}, x_{2 n+1}\right) \leq \omega_{1}\left(x_{2 n+1}, x_{2 n+2}\right),
$$

then

$$
M\left(x_{2 n}, x_{2 n+1}\right)=\omega_{1}\left(x_{2 n+1}, x_{2 n+2}\right)
$$

Thus

$$
\begin{aligned}
\psi_{1}\left(\omega_{1}\left(x_{2 n+1}, x_{2 n+2}\right)\right) & \leq \psi_{2}\left(\omega_{1}\left(x_{2 n+1}, x_{2 n+2}\right)\right)-\varphi\left(\omega_{1}\left(x_{2 n+1}, x_{2 n+2}\right)\right) \\
& <\psi_{1}\left(\omega_{1}\left(x_{2 n+1}, x_{2 n+2}\right)\right),
\end{aligned}
$$

a contradiction. Hence, for all $n \in \mathbb{N}, \omega_{1}\left(x_{2 n+1}, x_{2 n+2}\right)<\omega_{1}\left(x_{2 n}, x_{2 n+1}\right)$.

By the same argument, if we take, in the inequality (3.6), $x=x_{2 n-1}$ and $y=x_{2 n}$ we obtain

$$
\omega_{1}\left(x_{2 n}, x_{2 n+1}\right)<\omega_{1}\left(x_{2 n-1}, x_{2 n}\right), \text { for all } n \in \mathbb{N}^{*} .
$$

Then $\omega_{1}\left(x_{n+1}, x_{n+2}\right)<\omega_{1}\left(x_{n}, x_{n+1}\right)$, for all $n \in \mathbb{N}$. Thus, the sequence $\left\{\omega_{1}\left(x_{n}, x_{n+1}\right)\right\}$ is decreasing and bounded below. Therefore, it $\omega$-converges to some $r \geq 0$. By passing to upper limit in the inequality (3.7), we obtain

$\limsup _{n \rightarrow+\infty} \psi_{1}\left(\omega_{1}\left(x_{2 n+1}, x_{2 n+2}\right)\right) \leq \limsup _{n \rightarrow+\infty} \psi_{2}\left(M\left(x_{2 n}, x_{2 n+1}\right)\right)-\liminf _{n \rightarrow+\infty} \varphi\left(M\left(x_{2 n}, x_{2 n+1}\right)\right)$

Since

$$
\lim _{n \rightarrow+\infty} M\left(x_{2 n}, x_{2 n+1}\right)=\lim _{n \rightarrow+\infty} \max \left\{\omega_{1}\left(x_{2 n}, x_{2 n+1}\right), \omega_{1}\left(x_{2 n+1}, x_{2 n+2}\right)\right\}=r,
$$

and using the continuity of $\psi_{1}$ and $\psi_{2}$ and the lower semi-continuity of $\varphi$, we get $\psi_{1}(r) \leq \psi_{2}(r)-\varphi(r)$, which implies that $r=0$. Thus, $\lim _{n \rightarrow+\infty} \omega_{1}\left(x_{n}, x_{n+1}\right)=0$. Since $\omega$ is convex and satisfies the $\Delta_{2}$-condition and according to lemma 2.12, then $\left\{x_{n}\right\}$ is $\omega$-Cauchy sequence in $C$. Thus, from the completeness, $\left\{x_{n}\right\}$ is $\omega$-convergent to some $x \in C$.

If $T$ is $\omega$-continuous, then $\left\{x_{n+1}\right\}=\left\{T x_{n}\right\}$ is $\omega$-convergent to some $x$ and from the uniqueness of the limit we have $T x=x$.

If $\omega$ satisfies Fatou property, we have $\left.\omega_{1}(x, T x)\right) \leq \liminf _{n \rightarrow+\infty} \omega_{1}\left(x_{2 n+1}, T x\right)$. Following to the condition $(\mathrm{P}), x_{2 n} \preceq x$, for all $n \in \mathbb{N}$. Then

$$
\psi_{1}\left(\omega_{1}\left(x_{2 n+1}, T x\right)\right) \leq \psi_{2}\left(M\left(x_{2 n}, x\right)\right)-\varphi\left(M\left(x_{2 n}, x\right)\right)
$$


where

$M\left(x_{2 n}, x\right)=\max \left\{\omega_{1}\left(x_{2 n}, x\right), \omega_{1}\left(x_{2 n}, x_{2 n+1}\right), \omega_{1}(x, T x), \omega_{2}\left(x_{2 n}, T x\right)+\omega_{2}\left(x, x_{2 n+1}\right)\right\}$.

From

$$
\omega_{2}\left(x_{2 n}, T x\right) \leq \omega_{1}\left(x_{2 n}, x\right)+\omega_{1}(x, T x),
$$

we obtain

$$
\lim _{n \rightarrow+\infty} M\left(x_{2 n}, x\right)=\omega_{1}(x, T x) .
$$

Then

$$
\begin{aligned}
\psi_{1}\left(\omega_{1}(x, T x)\right) & \leq \psi_{1}\left(\liminf _{n \rightarrow+\infty} \omega_{1}\left(x_{2 n+1}, T x\right)\right) \\
& \leq \limsup _{n \rightarrow+\infty} \psi_{1}\left(\omega_{1}\left(x_{2 n+1}, T x\right)\right) \\
& \leq \limsup _{n \rightarrow+\infty}\left(\psi_{2}\left(M\left(x_{2 n}, x\right)\right)\right)-\liminf _{n \rightarrow+\infty}\left(\varphi\left(M\left(x_{2 n}, x\right)\right)\right) \\
& \leq \psi_{2}\left(\omega_{1}(x, T x)\right)-\varphi\left(\omega_{1}(x, T x)\right) .
\end{aligned}
$$

Which implies that $\omega_{1}(x, T x)=0$. The regularity insures that $T x=x$.

Now, let us prove that $S x=x$. If we take $y=x$ into the inequality (3.6), we obtain

$$
\psi_{1}\left(\omega_{1}(S x, x)\right) \leq \psi_{2}(M(x, x))-\varphi(M(x, x)) .
$$

Since $M(x, x)=\omega_{1}(S x, x)$, then

$$
\psi_{1}\left(\omega_{1}(S x, x)\right) \leq \psi_{2}\left(\omega_{1}(S x, x)\right)-\varphi\left(\omega_{1}(S x, x)\right),
$$

which implies that $\omega_{1}(S x, x)=0$. So, from the regularity of $\omega$, we conclude that $S x=x$.

Let us suppose that there exits another common fixed point $y$ of $T$ and $S$. If we assume that $\mathfrak{F}(T, S)$ is totally ordered, then $x$ and $y$ are comparable and according to (3.6), we have

$$
\psi_{1}\left(\omega_{1}(x, y)\right) \leq \psi_{2}\left(\omega_{1}(x, y)\right)-\varphi\left(\omega_{1}(x, y)\right),
$$

which insures that $\omega_{1}(x, y)=0$ and so $x=y$. Therefore, the uniqueness of the common fixed point of $S$ and $T$.

Remark 3.5. If we define $\omega$ by:

$$
\omega_{\lambda}(x, y)=\frac{d(x, y)}{\lambda}, \text { for all }(x, y) \in X^{2},
$$

we obtain Theorem 1.2 established by Abkar and Choudhury in [1].

EXAmple 3.6. Consider the space $X=[0,1]$ ordered by " $\preceq$ " which is the reverse of the usual order between the reals $(x \preceq y \Leftrightarrow x \geq y)$ and endowed with the modular metric defined for all $\lambda>0$ as follows:

$$
\omega_{\lambda}(x, y)=\left\{\begin{array}{lll}
\frac{x+y}{\lambda} & \text { if } & x \neq y \\
0 & \text { if } & x=y
\end{array}\right.
$$

Consider the two self-mappings $S$ and $T$ defined as follows: 


$$
S x=\frac{x}{4}, \text { for all } x \in[0,1] \text { and } T x=\left\{\begin{array}{lll}
\frac{x}{8} & \text { if } & 0 \leq x<1 \\
\frac{1}{2} & \text { if } & x=1
\end{array}\right.
$$

Consider the three functions defined for $t, s \in[0,+\infty[$ as follows:

$$
\psi_{1}(t)=t, \psi_{2}(t)=\frac{3 t}{4}, \varphi(t)=\frac{t}{8} .
$$

We can see that the functions $\psi_{1}, \psi_{2}$ and $\varphi$ satisfy all conditions described in the top of the section 3. It's easy to verify that

(i) $X_{\omega}=X$ is an $\omega$-complete modular metric space;

(ii) $\omega$ is convex and satisfies the $\Delta_{2}$-type condition and Fatou property;

(iii) $1 \preceq S 1 \preceq T S 1 \preceq S T S 1 \preceq(T S)^{2} 1 \preceq S(T S)^{2} 1 \preceq \ldots$

(iv) $(X, \preceq)$ satisfies the property $(\mathrm{P})$;

Let $x$ and $y$ be two comparable elements in $X$. Let us show that

$$
\psi_{1}\left(\omega_{1}(S x, T y)\right) \leq \psi_{2}(M(x, y))-\varphi(M(x, y)) .
$$

i.e,

$$
\omega_{1}\left(\frac{x}{4}, T y\right) \leq \frac{5}{8} M(x, y)
$$

Case.1. If $x=y=1$, then $\omega_{1}\left(\frac{1}{4}, \frac{1}{2}\right)=\frac{3}{4}$ and $M(1,1)=\frac{3}{2}$.

Case.2. If $x=1$ and $y \in[0,1[$, then

$$
\omega_{1}\left(\frac{1}{4}, \frac{y}{8}\right)=\frac{2+y}{8} \text { and } M(1, y)=\max \left\{1+y, \frac{5}{4}\right\} .
$$

- If $y<\frac{1}{4}, M(1, y)=\frac{5}{4}$.

- If $y \geq \frac{1}{4}, M(1, y)=1+y$.

Case.3. If $y=1$ and $x \in[0,1[$, then

$$
\omega_{1}\left(\frac{x}{4}, \frac{1}{2}\right)=\frac{2 x+4}{8} \text { and } M(x, 1)=\max \left\{1+x, \frac{3}{2}\right\} .
$$

- If $x<\frac{1}{2}, M(x, 1)=\frac{3}{2}$.

- If $x \geq \frac{1}{2}, M(x, 1)=1+x$.

Case.4. If $x, y \in\left[0,1\left[\right.\right.$ and $x=y$, then $\omega_{1}\left(\frac{x}{4}, \frac{y}{8}\right)=\frac{3 x}{8}$ and $M(x, y)=\frac{5 x}{4}$.

Case.5. If $x, y \in[0,1[$ and $x>y$, then

$$
\omega_{1}\left(\frac{x}{4}, \frac{y}{8}\right)=\frac{2 x+y}{8} \text { and } M(x, y)=\max \left\{\frac{5 x}{4}, x+y\right\} .
$$

- If $y \geq \frac{x}{4}$, then $M(x, y)=x+y$.

- If $y<\frac{x}{4}$, then $M(x, y)=\frac{5 x}{4}$.

Case.6. If $x, y \in\left[0,1\left[\right.\right.$ and $x<y$, then $M(x, y)=\max \left\{x+y, \frac{9 y}{8}\right\}$.

- If $x=\frac{y}{2}$, then $\omega_{1}\left(\frac{x}{4}, \frac{y}{8}\right)=0$.

- If $x \in] \frac{y}{8}, \frac{y}{2}[\cup] \frac{y}{2}, y\left[\right.$, then $\omega_{1}\left(\frac{x}{4}, \frac{y}{8}\right)=\frac{2 x+y}{8}$ and $M(x, y)=x+y$.

- If $x \leq \frac{y}{8}$, then $\omega_{1}\left(\frac{x}{4}, \frac{y}{8}\right)=\frac{2 x+y}{8}$ and $M(x, y)=\frac{9 y}{8}$.

One can easily see that (3.9) holds in all cases. Hence $S$ and $T$ verify all conditions of Theorem 3.4 and have a unique common fixed point which is 0 . 


\section{Conclusion}

The results in this paper,

(1) extend the work of Dutta and Choudhury in [11] from a metric to a partially ordered modular metric space.

(2) extend Theorem 2.2 of Abkar and Choudhury in [1] from a partially ordered metric space to a partially ordered modular metric space.

(3) extend Theorem 2.1 and Theorem 2.2 established by Harjani and Sadarangani in [13] from a metric space to a partially ordered modular metric space.

Remark 4.1. Recently, Jleli et al.[14] and Khamsi [15] replaced the $\Delta_{2}-$ condition by a weaker condition. One wonders if one can weaken the $\Delta_{2}-$ condition in the case of weak contractions defined on metric modular spaces.

\section{ACKNOWLEDGMENTS}

The authors wish to thank the editor and anonymous referees for their valuable suggestions and corrections.

\section{REFERENCES}

1. A.Abkar, B.S.Choudhury, Fixed point results in partially ordered metric spaces using weak contractive inequalities, Facta Universitatis(NIS), Ser. Math. Inform. Vol. 27, No 1 (2012), 1-11.

2. A.A.N.Abdou, Some fixed point theorems in modular metric spaces, J. Nonlinear Sci. Appl. 9 (2016), 4381-4387.

3. A.A.N.Abdou, M.A.Khamsi, Fixed point results of pointwise contractions in modular metric spaces, Fixed Point Theory and Applications 2013, 2013:163.

4. M.Abbas, S.Ali, P.Kumam, Common fixed points in partially ordered modular function spaces, Journal of Inequalities and Applications 2014, 2014:78.

5. Y.I.Alber, S.Guerre-Delabriere, Principles of weakly contractive maps in Hilbert spaces, new results in operator theory, In: Gohberg, I, Lyubich, Yu (eds.) Advances and Appl., vol. 98, pp. 7-22. Birkhauser Verlag, Basel (1997).

6. M. Beygmohammadi and A. Razani, Two fixed-point theorems for mappings satisfying a general contractive condition of integral type in the modular space, International Journal of Mathematics and Mathematical Sciences, Article ID 317107 (2010), 10 pages.

7. N. Cakic, Z.Kadelbur, S.Radenovic and A. Razani, Common fixed point results in cone metric spaces for a family of weakly compatible maps, Advances and Applications in Mathematical Sciences, Vol. 1 Issue 1 (2009), 183-207.

8. V.V.Chistyakov, Metric Modular Spaces-Theory and Applications, Springer International Publishing Switzerland 2015.

9. V.V.Chistyakov, Modular metric spaces, I: Basic concepts, Nonlinear Anal., 72 (2010), $1-14$.

10. L. Ćirić, A. Razan, S. Radivić and J.S. Ume, Common fixed point theorems for families of weakly compatible maps, Computers and Mathematics with Applications, 55 (2008), 2533-2543.

11. P.N.Dutta, B.S.Choudhury, A Generalisation of Contraction Principle in Metric Spaces, Fixed Point Theory and Applications. Volume 2008, Article ID 406368, 8 pages. 
12. M.B. Ghaemi and A. Razani, Fixed and periodic points in the probabilistic normed and metric spaces, Chaos, Solitons and Fractals, 28 (2006), 1181-1187.

13. J.Harjani, K.Sadarangani, Generalized contractions in partially ordered metric spaces and applications to ordinary differential equations, Nonlinear Analysis 72 (2010) 11881197.

14. M. Jleli, E. Karapinar and B. Samet, Best Proximity Point Result in Modular Spaces with the Fatou Property, Abstract and Applied Analysis, Volume 2013, Article ID 329451, 4 pages http://dx.doi.org/10.1155/2013/329451.

15. M.A. Khamsi, Quasicontraction Mappings in Modular Spaces without $\Delta_{2}-$ Condition, Fixed Point Theory and Applications, Volume 2008, Article ID 916187, 6 pages doi:10.1155/2008/916187.

16. M.A.Khamsi, W.M.Kozlowski, Fixed Point Theory in Modular Function Spaces, DOI 10.1007/978-3-319-14051-3, Springer International Publishing Switzerland 2015.

17. C.Mongkolkeha, P.Kumam, Some fixed point Results for Generalised Weak Contraction Mappings in Modular Spaces, International Journal of Analysis. Volume 2013, Articlle ID 247378, 6 pages.

18. M.Ozturk, M.Abbas, E.Girgin, Common fixed point results of a pair of generalized $(\psi, \varphi)$-contraction mappings in modular spaces, Fixed Point Theory and Applications (2016) 2016:19.

19. A.Padcharoen, D.Gopal, P.Chaipunya and P.Kumam, Fixed point and periodic point results for $\alpha$-type F-contractions in modular metric spaces, Fixed Point Theory and Applications (2016), 2016:39 DOI 10.1186/s13663-016-0525-4.

20. A. Razani, A fixed point theorem in the Menger probabilistic metric space, New Zealand J. Math., 35 (2006), 109-114.

21. A. Razani, Results in Fixed Point Theory, Andisheh Zarin publisher, Qazvin, August 2010.

22. A. Razani and M. Shiradaryazdi, Some results on fixed points in the fuzzy metric space, J. Appl. Math. Comput., 20 (2006), 401-408.

23. A. Razani and S. Homaeipour, Viscosity approximation to common fixed points of families of nonexpansive mappings with weakly contractive mappings, Fixed Point Theory Applications, Article ID 476913 (2010), 8 pages.

24. A. Razani and R. Moradi, Common fixed point theorems of integral type in modular spaces, Bulletin of the Iranian Mathematical Society, Vol. 35 No. 2 (2009), 11-24.

25. A. Razani and R. Moradi, Double sequence iterations for a strongly contractive mapping in the modular space, Iranian Journal of Mathematical Sciences and Informatics, 11 (2016), No. 2, 119-130.

26. A. Razani and V. Parvaneh, Some fixed point theorems for weakly T-Chatterjea and weakly T-Kannan-contractive mappings in complete metric spaces, Russian Mathematics (Iz. VUZ), 57 (2013), No.3, 38-45.

27. A. Razani and V. Parvaneh, On generalized weakly G-contractive mappings in partially ordered G-metric spaces, Abstract and Applied Analysis, Article ID 701910 (2012), 18 pages.

28. A. Razani and M. Samanipour, Common fixed point theorems for families of weakly compatible maps in a 2-metric space, Applied Mathematics and Information Sciences, $2(3)$ (2008), 275-289.

29. H. R. Sahebi, A. Razani, An explicit viscosity iterative algorithm for finding fixed points of two noncommutative nonexpansive mappings, Iranian Journal of Mathematical Sciences and Informatics, 11 (2016), No. 1, 69-83. 